\title{
Turbulence structure of non-uniform rough open channel flow
}

\author{
Priscilla Williams ${ }^{1}$, Vesselina Roussinova ${ }^{2, *}$, and Ram Balachandar ${ }^{1}$ \\ ${ }^{1}$ Civil and Environmental Engineering Department, 401 Sunset Ave, University of Windsor, Canada \\ ${ }^{2}$ MAME Department 401 Sunset Ave, University of Windsor, Canada
}

\begin{abstract}
This paper focuses on the turbulence structure in a non-uniform, gradually varied, sub-critical open channel flow (OCF) on a rough bed. The flow field is analysed under accelerating, near-uniform and decelerating conditions. Information for the flow and turbulence parameters was obtained at multiple sections and planes using two different techniques: twocomponent laser Doppler velocimetry (LDV) and particle image velocimetry (PIV). Different outer region velocity scaling methods were explored for evaluation of the local friction velocity. Analysis of the mean velocity profiles showed that the overlap layer exists for all flow cases. The outer layer of the decelerated velocity profile was strongly affected by the pressure gradient, where a large wake was noted. Due to the prevailing nature of the experimental setup it was found that the time-averaged flow quantities do not attained equilibrium conditions and the flow is spatially heterogeneous. The roughness generally increases the friction velocity and its effect was stronger than the effect of the pressure gradient. It was found that for the decelerated flow section over a rough bed, the mean flow and turbulence intensities were affected throughout the flow depth. The flow features presented in this study can be used to develop a model for simulating flow over a block ramp. The effect of the non-uniformity and roughness on turbulence intensities and Reynolds shear stresses was further investigated.
\end{abstract}

\section{Introduction}

The turbulence structure of a steady uniform open channel flow has been a subject of numerous experimental and numerical studies since the 1950s [1]. Due to change in the channel shape, size and slope, uniform flow conditions rarely occur in rivers and man-made channels. The changes of the bed topology coupled with the heterogeneous roughness create a complex non-uniform flow conditions which affect the flow resistance, sediment and pollution transport. Even in the simplest case of gradually varying open channel flow, the turbulence structure is significantly different from that in a uniform open channel flow [2]. The effect of non-uniformity in gradually varied flow is associated with the difference between the free surface and bed slope, $\Delta \mathrm{S}$. For $\Delta \mathrm{S}>0$ the flow is accelerating, while for $\Delta \mathrm{S}$ $<0$, the flow is decelerating and at $\Delta \mathrm{S}=0$, the flow is uniform. Accelerated turbulent

\footnotetext{
*Vesselina Roussinova: vtr@uwindsor.ca
} 
boundary layers tend to suppress the turbulence while decelerated turbulent boundary layers tend to promote turbulence due to the flow separation.

Many studies have investigated the effect of the pressure gradient in gradually accelerating and decelerating flows in open channels $[3,4,5]$. In contrast, the structure of non-uniform OCF flow over rough bed, which is more complicated, is not well researched. The presence of the pressure gradient is the main cause of non-uniformity and has a global influence on the flow. When the pressure gradient along the channel is near zero $(d p / d x \approx 0)$, uniform flow similar to a zero pressure gradient turbulent boundary layer can be achieved. The adverse pressure gradient (APG) or decelerating flow occurs when $d p / d x>0$. On the other hand, in the favorable pressure gradient (FPG) or accelerating flow, occurs with $d p / d x$ $<0$. Pressure gradient flows, both positive and negative, have been investigated for turbulent boundary layers, which provide helpful comparative information.

Non-uniform open channel flow over a rough bed is classified as a subset of turbulent boundary layer flow, which at high flow conditions is characterized by a high Reynolds number and the relative submergence conditions are such that flow will contain an inner (roughness) region and an outer region defined to include the overlap, wake and near-surface layers where viscous effects become small. The high Reynolds number and gradual nature of the flow expansion and contraction dictate that the turbulence structure of the flow maintains its general function in transferring potential and kinetic energy to dissipated heat as well as its general double-layer form [6]. The flow condition is also considered to be hydraulically rough which is indicated by a relatively high value for the roughness Reynolds number $\left(k^{+}=\right.$ $k \mathrm{u}_{\tau} / v>70$ ), where $k, u_{\tau}$ and $v$ are the roughness height, friction velocity and kinematic viscosity. Like turbulent boundary layers, the velocity distribution in non-uniform rough open channel flow can be divided into inner (roughness) and outer regions, which have two distinct sets of characteristic velocity and length scales, providing that the $k / d$ is sufficiently small, where $d$ is the flow submergence. In the roughness region, the friction velocity $u_{\tau}=$ $\left(\tau_{w} / \rho\right)^{1 / 2}$, is the appropriate velocity scale, and the characteristic length scale is $v / u_{\tau}$. Here, $\tau_{w}$ is the wall shear stress and $v$ is the kinematic viscosity of the fluid. Since the direct measurement of the wall stress is rather difficult on the rough bed, the standard approach used in turbulent boundary layers is to estimate friction velocity from the measured velocity distribution in the outer region. An accurate estimate of $u_{\tau}$ is required to investigate the effect of the imposed pressure gradient on the mean flow and turbulence quantities. Although $u_{\tau}$ is still considered to be an appropriate velocity scale in the outer layer, maximum velocity $\left(U_{e}\right)$ is used as velocity scale in the outer region in order to avoid bias due to the error in the calculation of the friction velocity. In addition, for the shallow open channel flow, the length scale in the outer region changes to be the depth of the flow $(d)$ instead of the boundary layer height $(\delta)$. The classical profile (smooth and rough) can be expressed as

$$
U^{+}=\frac{1}{\kappa} \ln y^{+}+A-\Delta U^{+}\left(k^{+}\right)+\frac{2 \Pi}{k} w\left(\frac{y}{\delta}\right)
$$

In Eq. 1, note that $\kappa=0.41$ is the von-Karman constant, the $y$ origin is located at the zeroplane displacement height $\varepsilon$ (so that the actual height from the bottom of the roughness is $y+\varepsilon)$ and the roughness height $k$ is measured from the bottom of the roughness elements to the top of the tallest ones. The roughness function $\Delta U^{+}$is zero for a smooth-wall flow and $\Pi$ is the Coles (1956) [7] wake parameter, which is typically 0.55. Experimental studies in uniform smooth OCF report different values for constant $A$ ranging from 5.0 to 5.5 [8], [9], [10]). Balachandar and Patel (2002) [11] among others have shown the validity of Eq. 1 for the overlap region $\left(30<y^{+}<0.2 d u_{\tau} / v\right)$. Far from the wall, the velocity distribution is not affected by the viscosity, while it is influenced by the free stream turbulence. In this region, a combination of two universal functions, the law of the wall and the law of the wake, can be 
used to describe the velocity profile. Krogstad et al. (1992) [12] proposed the following form of the defect law for smooth and rough wall turbulent boundary layers

$$
\frac{U_{e}-U}{u_{\tau}}=\frac{2 \Pi}{\kappa}\left[1-\frac{1}{2 \pi}\left((1-6 \Pi)\left(\frac{y+\varepsilon}{\delta}\right)^{2}-(1+4 \Pi)\left(\frac{y+\varepsilon}{\delta}\right)^{3}\right)\right]-\frac{1}{\kappa} \ln \left(\frac{y+\varepsilon}{\delta}\right)
$$

Here, $U_{e}$ is the maximum velocity and $\Pi$ is a wake parameter which strongly depends on the wall condition [13]. Cebeci and Smith (1974) [14] found experimentally that $\Pi$ increased monotonically with an increase of the Reynolds number in a zero-pressure gradient (ZPG) turbulent boundary layer. In addition, $\Pi$ depends strongly on the pressure gradient, with $\Pi$ increasing in the adverse pressure gradient flow and decreasing in the favorable pressure gradient flow. In ZPG rough turbulent boundary layers, however, there is also considerable evidence that the mean velocity profiles differ in the strength of the wake component. There are still considerable difficulties in accurate determination of all unknown parameters $\left(u_{\tau}, \varepsilon\right.$ and $\Pi$ ) in Eqs. 1 and 2 from the velocity measurements. These include the accurate determination of the wall stress and hence $u_{\tau}$, assessing whether spatial resolution of probes is adequate (especially near the wall), and determining the precise probe distance from the wall. The latter two difficulties become increasingly serious at large Reynolds number [15].

In this paper, the effects of non-uniformity on the mean velocity and turbulence quantities in open channel flow over a smooth and rough bed are investigated. Three connected beds with different slopes are used to maintain the effect of the non-uniformity on the turbulence characteristics of an open channel flow. The present velocity data were obtained using a two-component LDV and planar PIV techniques. The channel aspect ratio is large enough $(b / d=11)$, and the measurements are made in the center of the channel, so that the effect from secondary currents is reduced. The Reynolds number $\left(R e_{d}=d U_{e} / v\right)$ varied from 42,300 to 56,300. The measurements were obtained at low Froude numbers $(F r=0.26$ - 0.41) and there are no visible disturbances noticed on the free surface.

\section{Experiments}

The experiments were conducted in an open channel flume $10 \mathrm{~m}$ long and $1.23 \mathrm{~m}$ wide. The flume has acrylic sidewalls and bottom allowing optical access. The water supply system includes two pumps with flow control valves and a reservoir. A sluice gate located at the end of the flume was used to control and adjust the flow depth. In order to maintain non-uniform flows, the flume was equipped with a long smooth bed which consists of three distinct sections with different slopes. The first section had a positive bed slope of $+2^{\circ}$ to maintain the favorable pressure gradient flow. The second section was a flat wooden plate $(1.57 \mathrm{~m}$

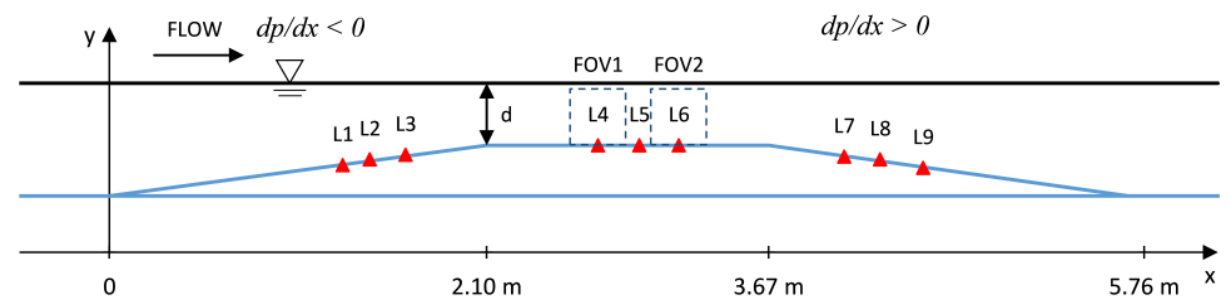

Figure 1. Side view schematics of the three connected ramps. The arrows point to the LDV measurement locations while the PIV vertical planes are indicated with the dashed squares. Not to scale. 
long) providing near uniform flow. The third section had a negative bed slope of $-2^{\circ}$, where decelerating flow was maintained. Figure 1 shows a schematic of the three ramps installed in the flume. For the rough bed, sand grains with $d_{50}=2.5 \mathrm{~mm}$ were glued to the three ramps. Velocity measurements using LDV were carried out at nine sections (three on each ramp) along the flume as shown in Figure 1. The details of the experiments are included in Table 1 where the measurement stations are located along the $\mathrm{x}$ axis and are referenced to the start of each bed slope.

Table 1. Summary of the experimental LDV conditions

\begin{tabular}{|c|c|c|c|c|c|}
\hline Flow conditions & Bed & Locations & $\begin{array}{l}\text { Distance from } \\
\text { the beginning } \\
\text { of the ramp } \\
\text { (cm) }\end{array}$ & Depth (m) & $\mathbf{R e}_{(\mathrm{d})}$ \\
\hline \multirow{3}{*}{$\begin{array}{c}\mathrm{FPG} \\
\mathrm{dp} / \mathrm{dx}<0\end{array}$} & \multirow{3}{*}{ Smooth/Rough } & L1 & 132 & 0.153 & 56000 \\
\hline & & L2 & 145 & 0.147 & 52700 \\
\hline & & L3 & 165 & 0.127 & 45500 \\
\hline \multirow{3}{*}{$\begin{array}{c}\mathrm{NZPG} \\
\mathrm{dp} / \mathrm{dx} \approx 0\end{array}$} & \multirow{3}{*}{ Smooth/Rough } & L4 & 62 & 0.110 & 42300 \\
\hline & & L5 & 97 & 0.110 & 49900 \\
\hline & & L6 & 107 & 0.110 & 53900 \\
\hline \multirow{3}{*}{$\begin{array}{c}\mathrm{APG} \\
\mathrm{dp} / \mathrm{dx}>0\end{array}$} & \multirow{3}{*}{ Smooth/Rough } & L7 & 42 & 0.130 & 51100 \\
\hline & & L8 & 62 & 0.143 & 56300 \\
\hline & & L9 & 86 & 0.153 & 56300 \\
\hline
\end{tabular}

In this study, two-component fiber-optic LDV system (DANTEC Dynamics) and a twodimensional PIV system (TSI Incorporated) were used to probe the flow. The LDV system was powered by a $300 \mathrm{~mW}$ Argon-Ion laser. The light scattered from the seeding particles was collected by a $500 \mathrm{~mm}$ focusing lens. The beam spacing was $38 \mathrm{~mm}$ and the beam half angle was $2^{\circ}$. At each measurement location, 10,000 validated samples were acquired while the system was operated at $0.01 \mathrm{~m}$ coincidence window. The data rate varied from $6 \mathrm{~Hz}$ near the bed to $250 \mathrm{~Hz}$ in the outer region. Prior to each experiment, the water was seeded with appropriate silver coated spherical glass particles with mean diameter of $10 \mu \mathrm{m}$ and specific gravity of 1.1 to facilitate velocity measurements. Details of the LDV configuration are avoided for brevity, and are available in Afzal et al., (2009) [16] and Roussinova et al. (2008) [17]. Due to the limitation of the transmitting optics, simultaneous two-component velocity measurements were available only for $85 \%$ of the flow depth. Velocity measurements were made in the central plane of the channel, where it is generally accepted that effect of the secondary flow is negligible. It should be noted that secondary flows are not expected to play any role since the channel aspect ratio $(b / h)$ is greater than five [18].

The PIV system consists of dual pulse Litron Nano L series Nd:YAG laser generating at $532 \mathrm{~nm}$ wavelength with an output energy of $135 \mathrm{~mJ} /$ pulse and maximum repetition rate of $15 \mathrm{~Hz}$. The laser sheet was formed through a $500 \mathrm{~mm}$ spherical lens and expanded through a $-15 \mathrm{~mm}$ cylindrical lens. The images were recorded using a TSI PowerViewPlus 4 MP 12bit digital camera with a resolution of $2048 \times 2048$ pixels operating in dual capture mode. A TSI PIV LaserPulse synchronizer was used to synchronize the camera's operation with the laser. The camera was mounted with a $70-300 \mathrm{~mm}$ Nikkor lens and adjusted to give the desired field-of-view. Two thousand image pairs were acquired at a frequency of $7.5 \mathrm{~Hz}$. The images were analyzed using a commercial software Insight $3 \mathrm{G}$ developed by TSI. The images were analyzed with a $64 \times 64$ pixel interrogation area and $50 \%$ overlap using a Fast Fourier 
Transform (FFT) correlator. The correlation peak was located within subpixel accuracy using a Gaussian curve-fitting method. This process yielded a final interrogation area with size of $32 \times 32$ pixels. The average field-of-view of $70 \times 70 \mathrm{~mm}^{2}$ and camera resolution of $2048 \times$ 2048 pixels was employed in the present PIV measurements corresponding to size of interrogation area of $2.2 \mathrm{~mm}$. Two fields of view are examined on the NZPG flow as indicated in Figure 1. The location of the profiles extracted from the PIV measurements matched with the LDV profiles at stations L4 and L6.

\section{Boundary layer characteristics}

The influence of the bed on flow in the inner layer is illustrated by variation of the friction velocity in the streamwise direction along the three ramps for smooth and rough bed. The values of the friction velocity were obtained by applying the Clauser chart method given by Eq. 1 for smooth bed experiments. Due to the increased uncertainty in determination of the zero-velocity location on the rough bed, $u_{\tau}$ values on the rough bed were obtained by extrapolating the Reynolds shear stress to the bed. Extrapolation of Reynolds stresses is the preferred method of estimating $u_{\tau}$ in turbulence research [18] accommodating availability of 2D multi-component LDV and PIV velocity measurements. For all experiments on the rough bed, the values of $u_{\tau}$ obtained from modified log-law and Reynolds shear stress methods compared well within the $\sim 15 \%$ of the experimental uncertainty. The variation of the friction velocity is shown in Figure 2 at all measurement locations, and the lines are included to better visualize the trend. The friction velocity on the smooth bed gradually increases over the three connected ramps which is consistent with the change of the pressure gradient. The trend is similar for the rough bed where the competing effects of the roughness and changing pressure gradient leads to further increase of the friction velocity. The present increasing trend of the friction velocity over the rough bed is consistent with the analysis of MacVicar and Obach (2015) [19] for the flow over riffle-pool gravel bed experiments.

The variation of the local mean velocity, $U$, normalized with $U_{e}$ at various vertical locations for smooth and rough flow are shown in Figure 3. As expected near the free surface at $y / d=0.8$, local mean velocity is maximum, and it is not affected by the change of the pressure gradient on the smooth bed. At the same vertical location, on the rough bed, however there is a notable reduction of the velocity due to the flow deceleration on the third ramp. In Figure $3 \mathrm{~b}$, the local mean velocity is reduced by about $30 \%$ throughout the flow depth due to the combined effect of the roughness and the adverse pressure gradient.

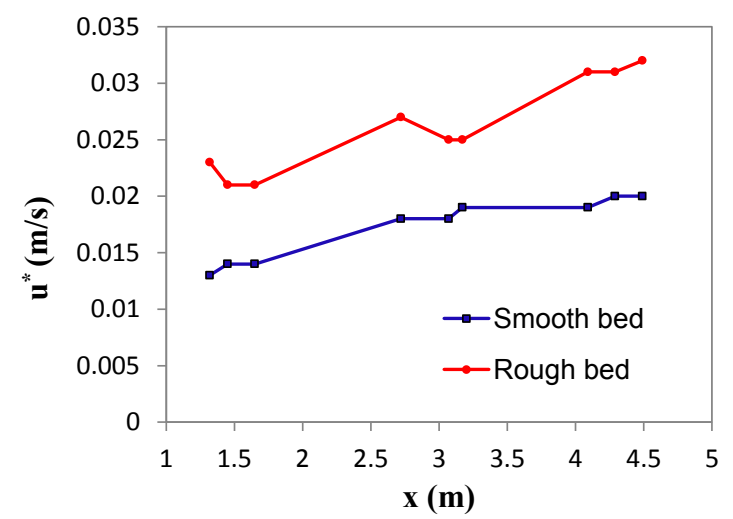

Figure 2. Variation of the friction velocity along the smooth and rough bed 
(a)

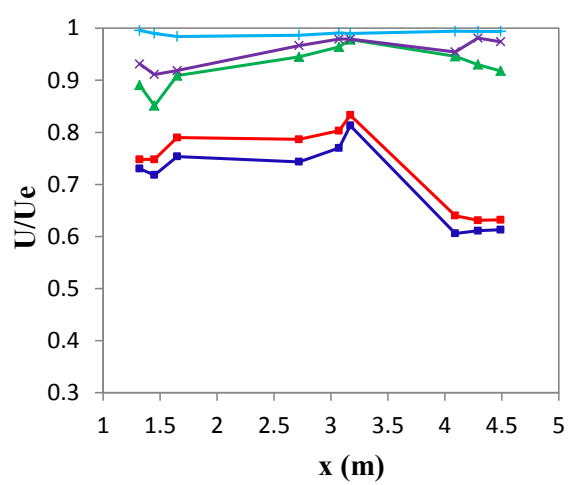

(b)

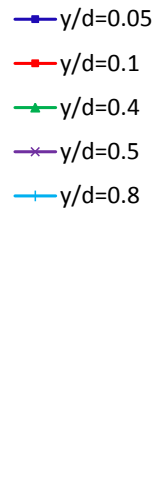

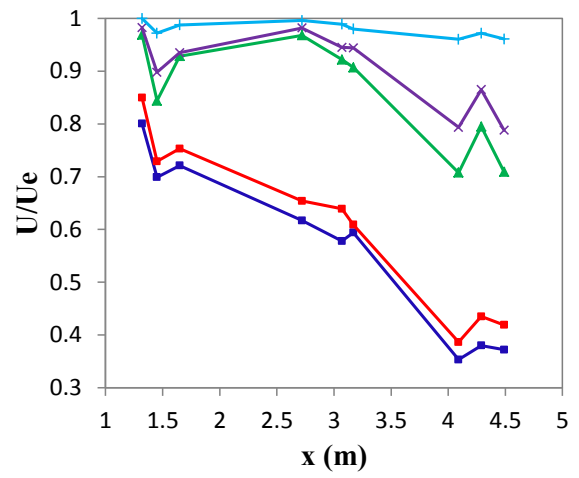

Figure 3. Variation of the local mean velocity $U / U e$ for a) smooth bed and b) rough bed.

\section{Mean flow and turbulence statistics on the flat rough bed}

Since the PIV measurements were available only on the flat rough bed, the mean flow and turbulence statistics are compared with the available LDV measurements at locations L4 and L6. In Figure 4, the mean velocity and $u_{r m s}$ profiles obtained from PIV and LDV techniques at locations L4 and L6 are shown. Note that in Figure 4a, the PIV measurements are restricted only to the outer region where they are in close agreement with the LDV measurements. Near the bed in the roughness region, the PIV measurements are affected by the light reflections which precludes extraction of accurate velocity measurements. In Figure $4 \mathrm{~b}, u_{r m s}$ profiles between LDV and PIV are in fair agreement despite some discrepancy observed in the outer layer.

To investigate the effect of the roughness in more detail, the mean velocity and turbulence parameters are compared with the smooth wall only for the NZPG flow conditions, where the pressure gradient is approximately zero and flow might be considered uniform. In Figure 5a, the mean velocity on the rough surface display a linear logarithmic region that is shifted by the roughness function, $\Delta U^{+}=9$, below the smooth profiles, indicating an increased momentum deficit. Friction velocity for the rough profiles was calculated using the classical Clauser method given by Eq. (1). Compared to the smooth wall,
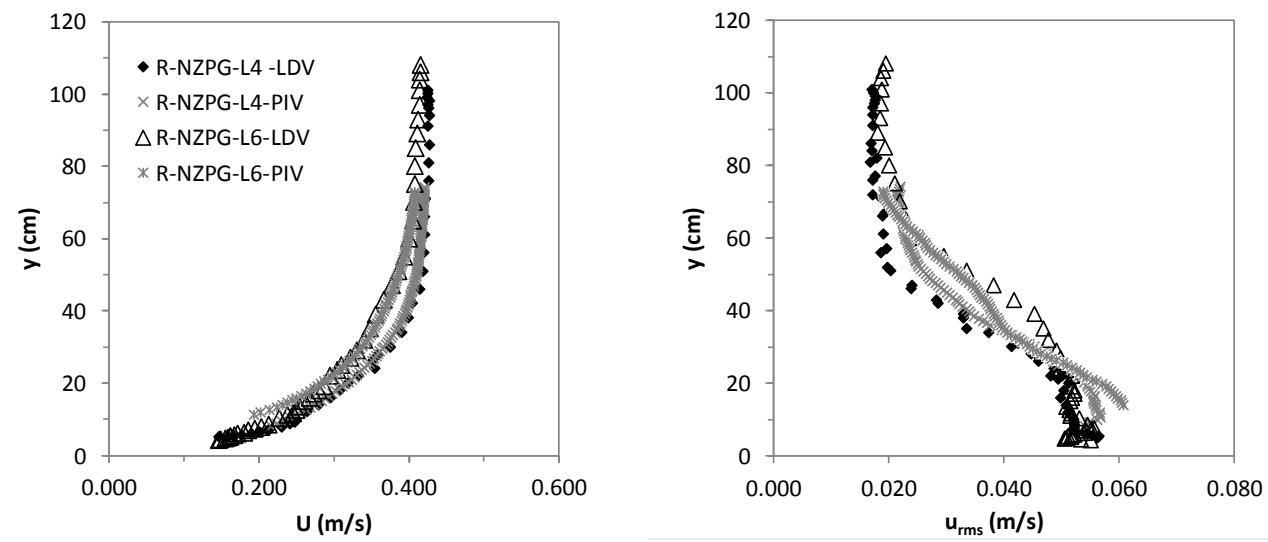

Figure 4. Comparison between the LDV and PIV profiles for NZPG flow at L4 and L6 over a rough bed. 
the rough profiles show a limited overlap region. Rough profiles at locations L5 and L6 follow the logarithmic law in the range $80<y^{+}<500$, while L4 profile has an extended overlap region which follows the logarithmic law in the range $40<y^{+}<500$. Local variations of the extent of the overlap layer can be attributed to the non-equilibrium flow conditions existing on the flat test section. The mean velocity profiles for all test cases are presented in outer scaling in Fig. 5b. Again, shown for comparison are the smooth wall profiles as well as the smooth profile of Roussinova et al., (2008) [17] for uniform flow using outer scaling with normalization by $U_{e}$ and $d$. The rough wall data show less full velocity profiles consistent with the effect of the roughness observed in the classical turbulent boundary layers. Under NZPG, the roughness Reynolds number $\left(k_{s}^{+}\right)$based on the roughness shifts $\left(\Delta U^{+}\right)$in the present study is greater than 70 , and thus, the flow is fully rough. While the smooth profiles collapse on to each other throughout the depth, the rough profile at L4 deviates from profiles at L5 and L6 in the outer region. As noted in Figure 4a, the flow at L4 tends to slightly accelerate resulting in change of the velocity profile. The differences are thought to be the result of the combined effect of the shallow depth and insufficient length of the flat ramp necessary for attaining self-similarity.

(a)

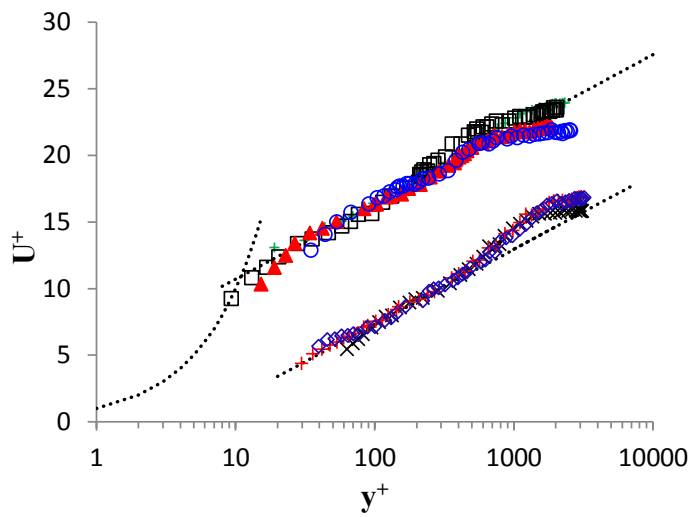

(b)

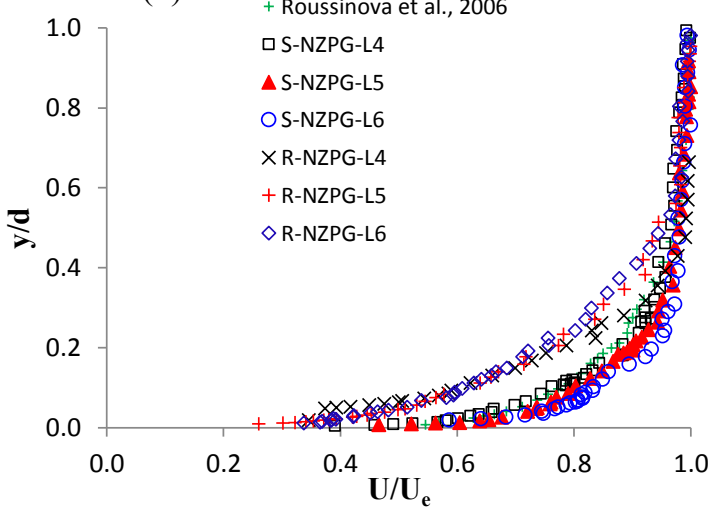

Figure 5. Inner log-low scaling (a) and outer (b) scaling of the velocity profiles for NZPG flow on smooth and rough bed.

In Figure 6, streamwise $u_{r m s}$ profiles for smooth and rough NZPG are shown normalized by the free stream velocity $\left(U_{e}\right)$ and flow depth $(d)$. As expected, $\mathrm{u}_{\mathrm{rms}}$ obtained on the rough wall are higher than that on the smooth wall throughout most of the overlap and outer layers for $\mathrm{y} / \mathrm{d}<0.6$. Consequently, the Reynolds shear stress is also much higher on the rough bed compared to the smooth bed as shown in Figure $6 \mathrm{~b}$.

\section{Conclusions}

The aim of the current study was to investigate the turbulence structure in a nonuniform, gradually varied, sub-critical open channel flow (OCF) on a rough bed. Flume experiments were carried out and two component LDV and planar PIV techniques are used to measure the velocity and turbulence parameters at various locations and planes on smooth and rough bed. Three types of flow were used to maintain non-uniform flow conditions: accelerating, decelerating, and near zero pressure gradient (flat plate). The change of the pressure gradient on the rough bed results in a strong feedback to the overlaying flow which precludes establishment of self-similarity. The local variation of the friction velocity is the highest for the decelerating flow on the rough bed where the turbulence is generated due to the increased 
strain rates.. These results are consistent with the reduction of the $U / U_{e}$ by $30 \%$ on the decelerating ramp for all vertical locations from $y / d=0.05$ to 0.80 . Further analysis of the mean velocity profiles confirms existence of the overlap layer. Moreover, for the NZPG test case the combined effects of the bed roughness and non-uniformity cause increase of both $u_{r m s}$ and $-\overline{u^{\prime} v^{\prime}}$ near the bed.
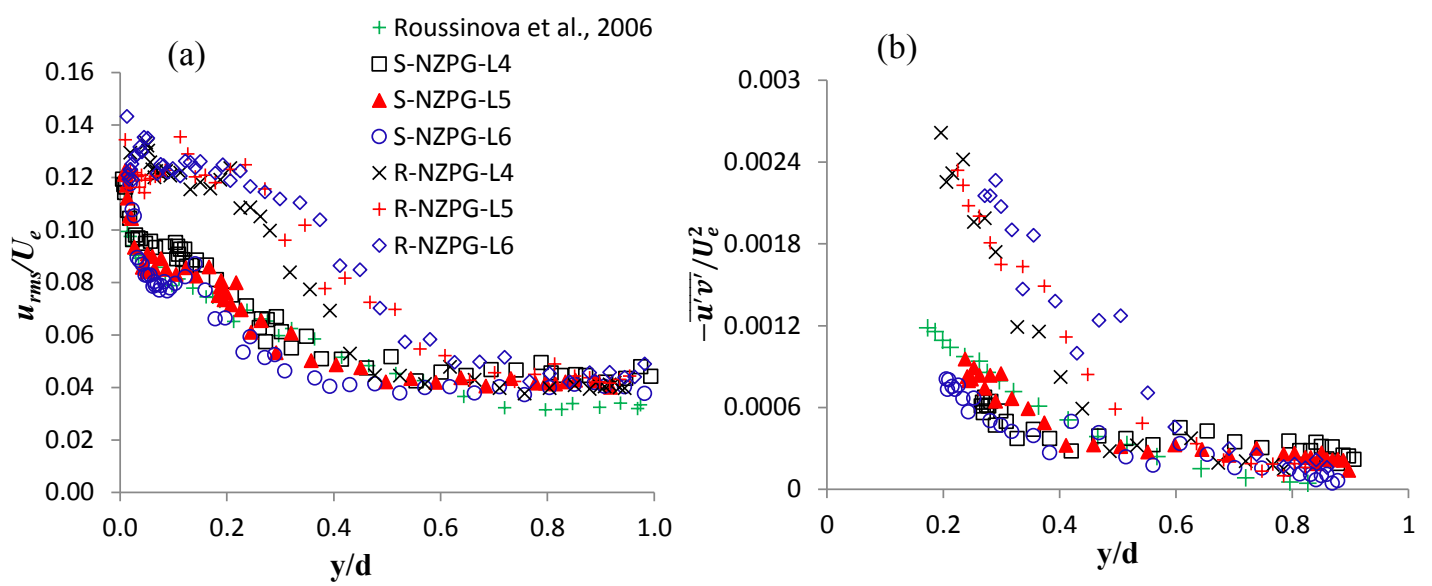

Figure 6. Outer scaling of $u_{r m s}$ profiles (a) and Reynolds shear stress profiles $-\overline{u^{\prime} v^{\prime}}$ (b) for smooth and rough NZPG flow

\section{References}

1. I. Nezu, J. Hydraul. Eng. 131(4), 229-246 (2005)

2. A.H. Cardoso, W.H. Graf, \& G. Gust, J. Hydraul. Res. 29(4), 525-543. (1991).

3. R. Balachandar, D. Blakely \& J. Bugg, Can. J. Civ. Eng. 29(2), 256-266 (2002)

4. T. Song \& Y.M. Chiew, J Eng. Mech. 127(3), 219-232. (2001)

5. A. Emamzadeh, Y. M. Chiew \&H. Afzalimehr, Adv. Water Res. 33, 271-283 (2010)

6. R. L. Stewart \& J.F. Fox, Adv. Water Res. 104, 271-283 (2017)

7. Coles, D, J. Fluid Mech. 1(2), 191-226 (1956)

8. F.H. Clauser, Adv. Appl. Mech. 4, 1-51 (1956)

9. I. Nezu \& W. Rodi, J. Hydraul. Eng. 112(5), 335-355 (1986)

10. P.M. Steffler, N. Rajaratnam \& A.W. Peterson, J. Hydraul. Eng. 111(1), 119-130. (1985)

11. R. Balachandar \& V.C. Patel, J. Hydraul. Eng. 128(10), 947-951 (2002)

12. P.A. Krogstad, R.A. Antonia, \& L.W.B. Browne, J. Fluid Mech. 245, 599-617 (1992)

13. M.F. Tachie, D.J. Bergstrom \& R. Balachandar, Exp. Fluids. 35(4), 338-346 (2003)

14. T. Cebeci \& A.M.O. Smith. Analysis of Turbulent Boundary Layers (Academic Press, Incorporated, 1974)

15. I.P. Castro, A. Segalini, \&P.H. Alfredsson J. Fluid Mech. 727, 119-313 (2013)

16. B. Afzal, M.A. Faruque \& R. Balachandar, J. Hydraul. Res. 47(1), 66-81 (2009)

17. V.T. Roussinova, N. Biswas \& R. Balachandar J. Hydr. Res, 46(SUPPL. 1), 36-48 (2008)

18. I. Nezu \& H. Nakagawa, Turbulence in open channels. IAHR/AIRH Monograph (Balkema, Rotterdam, The Netherlands, 1993)

19. B. MacVicar \& L. Obach, J. Hydraul. Eng. 141(11), 04015025 (2015) 